\title{
Elucidation of the how mechanism glial cells are actirated by the neural model cells of spinocerebellar ataxia type 21 in a cell death-independent manner
}

\author{
Tomoko Ohta ${ }^{1}$, Takahiro Seki ${ }^{1}$, Yuki Kurauchi ${ }^{1}$, Akinori Hisatsune ${ }^{2,3}$, Hiroshi Katsuki ${ }^{1}$ \\ ${ }^{I}$ Department of Chemico-Pharmacological Sciences, Graduate School of Pharmaceutical Sciences, Kumamoto \\ University, Japan, ${ }^{2}$ Priority Organization for Innovation and Excellence, Kumamoto University, Japan, ${ }^{3}$ Program for \\ Leading Graduation School HIGO Program, Kumamoto University, Japan
}

Spinocerebellar ataxia type 21 (SCA21) is an autosomal dominant neurodegenerative disease, characterized by early-onset and slowly progressive cerebellar ataxia, cognitive impairment and mental retardation. Recently, missense and nonsense mutations of transmembrane protein 240 (TMEM240) gene have been identified as the causative gene of SCA21. TMEM240 is assumed to be a transmembrane protein, but its localization and function remain unknown. We constructed SCA21 model mice with progressive motor dysfunction by the expression of mutant TMEM240 in cerebellar neurons using adeno-associated viral vectors. Immunohistchemical analyses revealed that the expression of mutant TMEM240 to cerebellar neurons did not induce neurodegeneration, but triggered the activation of astrocytes and microglia before the onset of motor dysfunction. In the present study, we attempted to elucidate the mechanism how SCA21 mutant TMEM240 activates glial cells without cell death using cultured cell line. Wild-type and mutant TMEM240 were transiently transfected to Neuro-2a cells, a mouse-derived neuroblastoma cell line, as a neuronal cell model. Since we assumed that some factors are released from neurons that express mutant TMEM240 and activate glial cells, we treated the culture media from Neuro-2a cells expressing TMEM240 to BV2 cells, a cell line derived from mouse microglia. Activation of BV2 cells was evaluated by mRNA levels of inflammatory factors, which were determined by RT-PCR analysis. Culture media from Neuro-2a cells expressing mutant TMEM240 significantly increased the mRNA of IL-1 $\beta$, an inflammatory cytokine, in BV2 cells. On the other hand, iNOS mRNA, at another inflammatory factor, was not affected. These findings suggest that some factors released from neurons expressing SCA21 mutant TMEM240 are involved in the activation of glial cells in a neurodegeneration-independent manner. 